\title{
FATORES DE RISCO PARA DOENÇAS CARDIOVASCULARES EM ADOLESCENTES OBESOS DE TRÊS DISTRITOS SANITÁRIOS DE GOIÂNIA
}

Silvia Salete Ozelame

Professora de Educação Física da Rede Municipal de Ensino da cidade de Goiânia.

Maria Sebastiana Silva

Professora Associada da Faculdade de Educação Física da Universidade Federal de Goiás.

\begin{abstract}
Resumo
O objetivo da pesquisa foi identificar e avaliar os riscos para doenças cardiovasculares em adolescentes obesos. Avaliou-se a circunferência muscular do braço, a dobra cutânea tricipital, perfil lipidêmico, glicemia, pressão arterial, nível de atividade física, doenças na família e hábito alimentar de 42 adolescentes, ambos os sexos, de 13 a 16 anos de idade. Os resultados indicaram que os triglicérides, colesterol, LDL-c e pressão arterial estavam elevados em 10\%, 30\%,30\% e 21\% dos adolescentes, respectivamente. Dentre eles, $36 \%$ eram pouco ativos, $64 \%$ sedentários e todos tinham hábito alimentar inadequado. Concluindo, os adolescentes avaliados apresentaram indicadores adicionais para doenças cardiovasculares e, portanto necessitam de cuidados especiais.
\end{abstract}

Palavras-chave: Adolescente - Obesidade - Doenças Cardiovasculares.

\section{Introdução}

obesidade é uma doença complexa e multifatorial que envolve a interação de
influências metabólicas, fisiológicas, comportamentais e sociais. Entre os fatores ambientais, os destaques são para os hábitos alimentares inadequados e o sedentarismo. Ela está associada ao desenvolvimento de diversas doenças crônicas não transmissíveis, como por exemplo, a hipertensão arterial, hipercolesterolemia, hiperlipidemia, doenças coronarianas entre outras (DÂMASO, 2003; GUEDES et al., 2006).

Quanto à prevalência de obesidade em indivíduos jovens brasileiros, estudos realizados na final da década de 80 apontavam índices de 2,5 a 8\% em crianças de famílias de baixo e alto poder aquisitivo, respectivamente (BRASIL, 1991). Outro estudo realizado em 2003 apontou prevalência de 9,3\% para obesidade e de 3,0\% para sobrepeso, tanto em adolescentes da região Nordeste como Sudeste (ABRANTES; LAMOUNIER; COLOSIMO, 2003). Silva, Balaban e Motta (2005), estudando o perfil de adolescentes encontraram inversão nos índices de prevalência de sobrepeso e obesidade, com valores de $8,8 \%$ e $4,9 \%$, respectivamente.

A literatura tem apresentado fortes evidências referentes à qualidade de vida das diferentes populações com sobrepeso e obesidade, apontando uma sensível diminuição na expectativa de vida dos indivíduos que convivem com a obesidade por períodos prolongados (FONTAINE et al., 2003). Além disso, o impacto ocasionado, por estas patologias, tem sido significativo em diversos setores da sociedade. No que se 
referem ao setor financeiro, estudos têm indicado que gastos com obesidade e doenças relacionadas, têm aumentado de forma preocupante nos últimos anos (WANG; MONTEIRO; POPKIN, 2002). Considerando os altos índices de obesidade que atingem a população de adolescentes e os riscos para saúde, o objetivo deste trabalho foi identificar os riscos para doenças cardiovasculares em adolescentes obesos e préobesos atendidos em três distritos sanitários da cidade de Goiânia.

\section{Métodos}

Este trabalho trata-se de um estudo transversal de caráter observacional, aprovado pelo Comitê de Ética em Pesquisa do Hospital das Clínicas - HC/UFG (Processo $\mathrm{n}^{\circ}$ 170/2006), realizado com 42 adolescentes obesos integrantes de três distritos sanitários situados na região central da cidade de Goiânia-GO.

Os critérios de inclusão para participação na pesquisa foram: adolescentes com idade entre 12 e 18 anos e índice de massa corporal acima do percentil 85 e disposição em participar das avaliações antropométricas e nutricionais. Os adolescentes que aceitaram participar da pesquisa tiveram os termos de consentimentos livres e esclarecidos assinados pelos seus responsáveis legais.

Os indicadores adicionais do risco para doenças cardiovasculares, nos adolescentes, foram determinados a partir de informações da composição corporal, alguns parâmetros bioquímicos, pressão arterial, nível de atividade física e hábito alimentar.

A composição corporal foi avaliada pelo índice de massa corporal (IMC), a circunferência do braço (CB), a dobra cutânea tricipital (TR) e a circunferência muscular do braço (CMB). Mensurações da massa corporal $(\mathrm{kg})$ e da estatura $\left(\mathrm{m}^{2}\right)$ foram realizadas para o cálculo do IMC (GARROW; WEBSTER, 1985). Para análise deste parâmetro utilizaram-se as curvas de referência do National Center for Health Statistics (NCHS, 2002), considerando-se pré-obesidade quando o percentil for maior que 85 e obesidade para percentil acima de 95 . Para medir a CB foi utilizada fita métrica não extensível e para dobra TR, plicômetro Cescorf, pressão constante de $10 \mathrm{~g} / \mathrm{mm} 2$ e precisão de um milímetro. $\mathrm{A} C \mathrm{CMB}$ foi obtida a partir da fórmula: $\mathrm{CMB}=$ $\mathrm{CB}-3,14 \mathrm{x}$ TR. Tanto a CMB, como CB e dobra TR foram avaliadas considerandose os valores em percentil para sexo e idade descritos por Frisancho (1990). Os pontos de corte inferior e superior de normalidade para $\mathrm{CMB}$ e $\mathrm{CB}$ foram os percentis $10 \mathrm{e}$ 90. Para a dobra TR, os percentis de 15 e 85 , respectivamente.

Os parâmetros bioquímicos avaliados foram: glicemia, triglicérides, colesterol total e frações lipídicas (HDL-c e LDL-c). A coleta de sangue e as análises foram realizadas em laboratórios da rede pública de saúde. Todos estavam em jejum de 12 horas, conforme instrução dos laboratórios. Os valores bioquímicos fornecidos pelos laboratórios foram avaliados conforme a especificação para idade em níveis desejáveis, limítrofes e aumentados (SANTOS et al, 2004).

A medida da pressão arterial foi obtida em ambiente calmo, com o adolescente sentado, após três a cinco minutos de repouso e com a fossa cubital direita apoiada na altura do coração. Foram realizadas duas medidas da pressão arterial, tanto da sistólica quanto da diastólica, para estimar a média da pressão aferida naquele momento. A pressão arterial foi obtida em aparelho de pressão digital automático de braço, Marca BD, com manguito de tamanho apropriado à circunferência do braço dos adolescentes. Os valores 
foram comparados a valores normativos específicos de idade, sexo e percentil de estatura, descritos pela V Diretriz Brasileira de Hipertensão Arterial (MION JUNIOR, 2006).

Para avaliar o nível de atividade física dos adolescentes aplicou-se o Questionário Internacional de Atividades Físicas para adolescentes (CELAFISCS, 2005). Foram questionadas informações sobre as atividades físicas realizadas nas aulas de Educação Física na escola; atividades esportivas, outras atividades diárias e as atividades físicas no lazer. Para classificação do nível de atividade física utilizaram-se os critérios disponíveis no Celafics (2006).

Para classificar o hábito alimentar foi utilizado o Questionário Semiquantitativo de Freqüência Alimentar validado (SLATER et al., 2003). Os dados do questionário foram avaliados de duas maneiras, na primeira considerou-se como alimento integrante do hábito alimentar aquele consumido pelo menos uma vez por semana pelos adolescentes (OLIVEIRA; THÉBAUD-MONY, 1998) e a segunda, avaliou o número porções de alimentos consumidos com base nas porções recomendadas na pirâmide alimentar adaptada para a população brasileira (PHILIPPI et al., 1999).

Também se investigou o histórico familiar de doenças associadas à obesidade e alguns parâmetros do nível socioeconômico dos pais ou responsáveis legais. Para a história familiar de doenças, considerou-se, nos pais e avós, a presença diagnosticada de pelo menos uma das seguintes patologias: diabetes, hipertensão, dislipidemia, doenças cardiovasculares e câncer. Como parâmetros socioeconômicos foram considerados o grau de escolaridade e a renda familiar.

\section{Resultados}

Dos 42 adolescentes classificados obesos, que aceitaram participar da pesquisa, $50 \%$ eram do sexo masculino e 50\% do feminino. A média de idade foi de 13,79 \pm 1,12 anos e todos apresentavam valores de IMC igual ou acima do percentil 90.

Os valores médios da composição corporal e dos índices bioquímicos dos adolescentes estão descritos na Tabela 1 . Em relação aos valores de circunferência do braço, aproximadamente $76 \%$ apresentaram valores de risco e os restantes localizavam-se no percentil 90, limite máximo de normalidade. Para os valores de dobra cutânea triciptal, que representa a quantidade de gordura armazenada no braço, todos os adolescentes apresentaram níveis de obesidade. Quanto à circunferência muscular do braço, aproximadamente $57 \%$ apresentaram valores normais e dos $43 \%$ restantes, metade estava em risco e os demais se encontravam no percentil 90, limite máximo de normalidade.

Tabela 1 - Valores médios e desvio padrão dos parâmetros antropométricos e bioquímicos detectados nos adolescentes obesos e com sobrepeso

\begin{tabular}{|c|c|c|}
\hline Variáveis & Valores médios & Desvio padrão \\
\hline Estatura $(\mathbf{c m})$ & 163,68 & 9,13 \\
\hline Peso $(\mathbf{k g})$ & 79,94 & 21,44 \\
\hline IMC $\mathbf{( k g} / \mathbf{m} \mathbf{2})$ & 29,39 & 4,51 \\
\hline CB $\mathbf{( c m})$ & 328,57 & 39,90 \\
\hline TR $(\mathbf{m m})$ & 34,49 & 5,33 \\
\hline
\end{tabular}

(continua) 
(continuação)

\begin{tabular}{|c|c|c|}
\hline Variáveis & Valores médios & Desvio padrão \\
\hline CMB (cm) & 220,28 & 40,17 \\
\hline Glicemia (mg/dL) & 76,67 & 6,36 \\
\hline Triglicérides (mg/dL) & 81,56 & 19,84 \\
\hline Colesterol total (mg/dL) & 155,89 & 22,36 \\
\hline HDL-c (mg/dL) & 41,67 & 3,24 \\
\hline LDL-c (mg/dL) & 97,91 & 21,43 \\
\hline
\end{tabular}

$I M C$ - indice massa corporal; $C B$ - circunferência do braço; TR - dobra cutânea tricipital; $C M B$ - circunferência do braço; $H D L-c$ - high density lipoprotein; $L D L-c$ - low density lipoprotein.

$\mathrm{Na}$ Figura 1 estão representados os valores comparativos do perfil lipidêmico com o IMC e dos níveis da pressão arterial com IMC, de todos os adolescentes avaliados.

Quanto aos dados perfil lipidêmico, a maioria (cerca de 90\%) apresentou valores normais de triglicérides, porém, o colesterol total estava com valores considerados limítrofes para 20\% e aumentado para 10\% dos adolescentes. Quanto ao LDL-c foi considerado adequado para $60 \%$ da amostra, alto para $20 \%$ e, limítrofe para os demais.

A pressão arterial sistólica e diastólica (Figura 1) dos adolescentes foi comparada com os valores de referência preconizados pela V Diretrizes Brasileiras de Hipertensão Arterial (MION JUNIOR, 2006) e indicaram que aproximadamente $21 \%$ dos adolescentes eram hipertensos e aproximadamente $37 \%$ apresentavam valores classificados como limítrofes, os demais tinham pressão arterial considerada adequada. Os valores médios de pressão sistólica foram $117,73 \pm 12,23$ e diastólica $68,27 \pm 10,81$.

Quanto ao nível de atividade física dos adolescentes obesos atendidos nos três distritos sanitários, $36 \%$ eram pouco ativos e $64 \%$ eram sedentários.

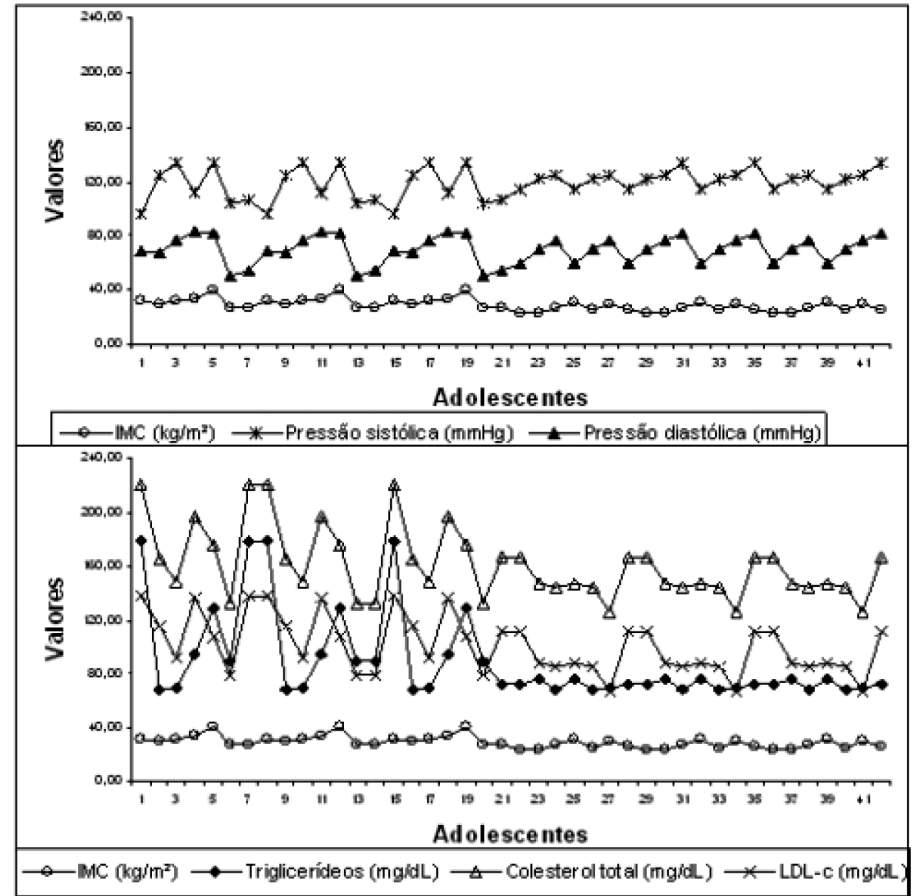

Figura 1. Representação comparativa entre pressão arterial sistólica e diastólica com o IMC (A) e do perfil lipidêmico com o IMC (B), dos adolescentes obesos. (IMC - índice de massa corporal e LDL-c - lipoproteína de baixa densidade) 
Os dados referentes ao hábito alimentar dos adolescentes indicaram que a maioria consumia uma grande quantidade de açúcar, doce, balas, chocolate, refrigerantes, sorvete e biscoito com recheio. Entre as frutas e vegetais, os de consumo habitual foram banana, laranja, tomate e alface. Quanto ao consumo de leite e derivados, apenas o leite integral e o iogurte foram considerados hábitos alimentares dos adolescentes. Outro alimento consumido habitualmente por todos adolescentes foi o feijão. Além destes, foi relatado alta freqüência de consumo semanal de salgados assados e fritos, misto quente, sanduíche natural, lingüiça, maionese, margarina, pão de queijo e batata tipo chips e salgadinhos.

Quanto à adequação das porções consumidas de alimentos de cada grupo (Tabela 2), mais de 78\% dos adolescentes consumiam porções de doces muito superiores às recomendadas. Entre os alimentos menos consumidos destacaram-se as hortaliças, frutas e carnes.

Ainda em relação aos hábitos alimentares, foi observado que a maioria (71\%) dos adolescentes não fazia o desjejum. A justificativa era porque estudavam pela manhã e saiam muito cedo de casa, não tendo tempo para comer. Dessa forma, esta refeição era substituída por um lanche altamente calórico, no horário do lanche, na escola.

Tabela 2 - Distribuição dos adolescentes obesos de acordo com a classificação1 de consumo das porções de cada grupo alimentar.

\begin{tabular}{|c|c|c|c|c|c|c|}
\hline \multicolumn{2}{|c|}{ Grupo de Alimentos } & \multicolumn{5}{c|}{ Classificação } \\
\cline { 2 - 7 } \multicolumn{2}{|c|}{} & Abaixo & $\begin{array}{c}\text { Adequa- } \\
\text { do }\end{array}$ & Acima & Não consome & Total \\
\hline \multirow{2}{*}{ Cereais/pães } & $\mathrm{n}$ & 24 & 15 & 3 & 0 & 42 \\
\cline { 2 - 8 } & $\%$ & 57,2 & 35,7 & 7,1 & 0 & 100 \\
\hline \multirow{2}{*}{ Hortaliças } & $\mathrm{n}$ & 27 & - & - & 15 & 42 \\
\cline { 2 - 8 } & $\%$ & 64,3 & - & - & 35,7 & 100 \\
\hline \multirow{3}{*}{ Frutas } & $\mathrm{n}$ & 3 & 9 & 6 & 24 & 42 \\
\cline { 2 - 8 } & $\%$ & 7,1 & 21,4 & 14,3 & 57,2 & 100 \\
\hline \multirow{3}{*}{ Leite e derivados } & $\mathrm{n}$ & 33 & - & - & 9 & 42 \\
\cline { 2 - 8 } & $\%$ & 78,6 & - & - & 21,4 & 100 \\
\hline \multirow{2}{*}{ Carnes e derivados } & $\mathrm{n}$ & - & 12 & & 30 & 42 \\
\cline { 2 - 8 } & $\%$ & - & 28,6 & & 71,4 & 100 \\
\hline \multirow{2}{*}{ Feijão } & $\mathrm{n}$ & 3 & 27 & 6 & 6 & 42 \\
\cline { 2 - 8 } & $\%$ & 7,1 & 64,3 & 14,3 & 14,3 & 100 \\
\hline \multirow{2}{*}{ Doces } & $\mathrm{n}$ & - & 6 & 33 & 3 & 42 \\
\cline { 2 - 8 } & $\%$ & - & 14,3 & 78,6 & 7,1 & 100 \\
\hline \multirow{2}{*}{ Óleos } & $\mathrm{n}$ & - & 36 & 6 & - & 42 \\
\hline & $\%$ & - & 85,7 & 14,3 & - & 100 \\
\hline
\end{tabular}

1 Classificação conforme recomendação da Pirâmide Alimentar adaptada para população brasileira. 
Quanto ao histórico de doenças na família (Figura 2), as patologias citadas foram diabetes, hipertensão, hipercolesterolemia, problemas cardíacos e câncer. Vale ressaltar, que o diabetes foi a doença presente, em pelo menos um membro da família, de todos os adolescentes investigados.

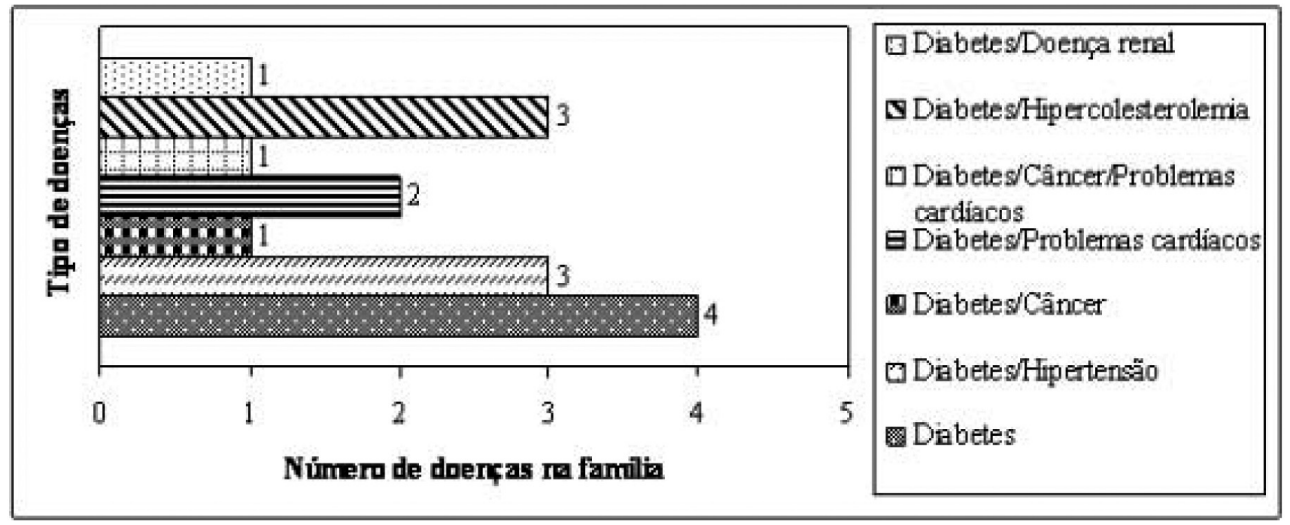

Figura 2. Doenças presentes em indivíduos da família dos adolescentes obesos.

No que se refere ao grau de escolaridade dos pais ou responsável legal pelo adolescente, $71 \%$ eram analfabetos ou tinham o ensino fundamental incompleto. A renda familiar variou de 1,5 a 6,5 salários mínimos sendo que $36 \%$ recebiam de 1,5 a 2,5; $28,5 \%$ de 2,5 a 4,$5 ; 28,5 \%$ de 4,6 a 6,5 e $7 \%$ não souberam informar.

\section{Discussões}

Os adolescentes obesos atendidos nos três distritos sanitários de Goiânia apresentaram valores médios de estatura semelhantes aos que participaram do Programa de Atendimento Interdisciplinar ao Adolescente Obeso do Centro de Práticas Esportivas da Universidade de São Paulo (CEPEUSP), enquanto que os valores de IMC foram menores. $\mathrm{O}$ valor médio de estatura dos adolescentes do CEPEUSP foi de $161,7 \pm 6,9 \mathrm{~cm}$ e o de IMC foi de $33,5 \pm 5,2 \mathrm{~kg} / \mathrm{m} 2$ (DENADAI et al., 1998).

Em se tratando de métodos antropométricos para avaliar a obesidade, as medidas de IMC e a espessura da dobra cutânea tricipital são bem aceitos por pesquisadores da área de saúde (SARDINHA et al., 1999; GIUGLIANO; MELO, 2004), por serem de baixo custo e de fácil aplicação. Estudo realizado em escolares obesos encontrou boas correlações entre as variáveis IMC e TR, o mesmo pôde ser sugerido neste estudo, visto que, o percentual de indivíduos obesos foi igual quando classificados pelas duas variáveis mencionadas (FERNANDES et al, 2007).

Comparando-se o perfil lipídico dos adolescentes deste estudo com o de Stabelini Neto (2007), foi possível observar valores semelhantes de triglicérides $(82,54 \mathrm{mg} / \mathrm{dL})$, maiores de colesterol total $(140,17 \mathrm{mg} / \mathrm{dL})$ e de HDL-c $(45,86 \mathrm{mg} / \mathrm{dL})$ e menores de LDL-c (77,20 mg/dL). Já os valores relatados por Ribeiro et al. (2006) foram maiores para colesterol total $(158,02 \mathrm{mg} / \mathrm{dL})$, HDL-c $(46,45)$ e LDL-c $(93,86 \mathrm{mg} / \mathrm{dL})$.

O aumento nas concentrações de LDL-c e colesterol total (CT) estão relacionados ao risco aumentado de doenças cardiovasculares, ao contrário das concentrações 
de HDL, que atuam como um fator de proteção para estas doenças. Quanto maior a concentração de LDL, maior sua facilidade de penetrar no endotélio vascular. A LDL é capaz de passar pela parede endotelial, penetrar na parede da artéria e sofrer oxidação na camada íntima desta. A conseqüência disto é a formação de placas de ateroma e o desenvolvimento de doenças cardiovasculares (KRAUSS, 2004). Em geral, $1 \%$ de diminuição nos níveis de LDL está associado a uma redução de 2-3\% no risco de desenvolvimento de doenças cardiovasculares (SANTOS, 2001). Além disso, existem relações entre variações no perfil lipídico e índice de massa corporal, sedentarismo, ingestão de álcool, uso de anticoncepcionais e antecedentes familiares de acidente vascular cerebral (COELHO et al., 2005).

A hipertensão arterial apresenta alta prevalência na população brasileira e influencia o surgimento de doenças cerebrais, coronarianas, insuficiência cardíaca, insuficiência renal crônica e doenças vasculares (MION JUNIOR, 2006). Alguns estudos têm relatado altos índices de hipertensão em populações jovens, principalmente entre os obesos. Ribeiro et al. (2006) em seu estudo sobre fatores adicionais de risco para doenças cardiovasculares, associadas ao excesso de peso em crianças e adolescentes, encontrou valores médios de $113,00 \pm 0,50 \mathrm{mmHg}$ e de $70,00 \pm 0,4 \mathrm{mmHg}$ para a pressão sistólica e diastólica, respectivamente, enquanto a pressão sistólica dos adolescentes do NECASA/HC, foi superior. Araújo et al. (2008) estudando os fatores de risco para hipertensão, em adolescentes, relataram índices aproximados de 109 $\mathrm{mmHg}$ para pressão sistólica e de $73 \mathrm{mmHg}$ para diastólica. Tanto os valores deste estudo como os de Ribeiro et al. (2006) e de Araújo et al. (2008) foram superiores aos encontrados por Stabelini Neto (2007), no qual os valores relatados foram 94,22 mmHg para pressão sistólica e 68,52 $\mathrm{mmHg}$ para diastólica.

As dislipidemias, juntamente com a hipertensão, o diabetes e o hábito de fumar, são consideradas fatores de risco primários para o desenvolvimento de doenças cardiovasculares (AMERICAN DIABETES ASSOCIATION, 2003). Além disso, fatores secundários, como o gênero, a idade e a falta de exercícios físicos aumentam o risco de desenvolvimento destas complicações (PITANGA; LESSA, 2005; LESSA, 2004). Vale destacar que os adolescentes deste estudo eram pouco ativos ou sedentários, sendo este um fator adicional para as doenças cardiovasculares. Guedes et al. (2006) estudando fatores de risco para doenças cardiovasculares em adolescentes constataram que aproximadamente $16 \%$ deles apresentam pelo menos um fator de risco biológico para o desenvolvimento dessas doenças. Ainda verificaram que o sobrepeso estava associado à ingestão excessiva de gorduras e que a pressão arterial elevada estava relacionada ao estilo de vida sedentário e ao uso de tabaco.

Estudos relacionados ao consumo alimentar de adolescentes têm indicado baixa ingestão de produtos lácteos, frutas, hortaliças, alimentos fontes de proteína e ferro, concomitantemente, ao elevado consumo de açúcar e gordura (VIEIRA et al., 2002; VIEIRA et al., 2005). Neste estudo, os resultados foram semelhantes, as frutas, legumes e hortaliças também não faziam parte do consumo habitual dos adolescentes avaliados, demonstrando que o padrão alimentar qualitativo necessita de ajustes para prevenir deficiências nutricionais específicas que podem repercutir na saúde e na composição corporal. 
Em relação aos hábitos alimentares, estudo realizado com adolescentes recémingressos na universidade encontrou que 57,3\% realizavam uma das refeições principais e $37 \%$ ingeriam alimentos doces ou gordurosos todos os dias. Entre os alimentos mais rejeitados destacaram-se os do grupo das hortaliças e as frutas (TOJO et al., 1995). É possível que esses alimentos estejam sendo substituídos por refrigerantes, salgadinhos e biscoitos (VIEIRA, 2002).

$\mathrm{Na}$ população brasileira, o feijão é considerado alimento de consumo habitual. Ressalta-se a importância do seu consumo juntamente com o de arroz, fornecendo aminoácidos como lisina, treonina, metionina e triptofano, importante aporte protéico vegetal, principalmente quando a proteína animal não faz parte do consumo habitual (VIEIRA, 2005). No presente estudo, o feijão foi o alimento mais consumido, entretanto, existe a possibilidade de que a biodisponibilidade do feijão com relação ao ferro esteja comprometida, uma vez que foi detectada, também neste estudo, a baixa freqüência do consumo de frutas cítricas, fonte importante de vitamina $\mathrm{C}$.

A redução indiscriminada no consumo dos grupos de alimentos base da alimentação, como os cereais, ou fonte de importantes nutrientes, como o leite, as carnes e as frutas, é contra-indicada para qualquer parcela da população, sobretudo para os adolescentes que estão em fase de crescimento e desenvolvimento acelerado (TOJO et al., 1995). Reforça-se, assim, a necessidade de medidas educativas específicas para esse grupo etário, de maneira a transmitir informações corretas, desmistificando concepções a respeito da alimentação e alertando-os acerca dos riscos nutricionais por carência e excesso de nutrientes e energia.

A crescente atenção dedicada a essas questões pode ser exemplificada pela criação de programas de educação envolvendo, inclusive, os meios de comunicação de massa. Conforme informações da Declaração de Consenso da Interamerican Heart Foundation (1998) sobre a saúde cardiovascular de crianças e jovens, as doenças cardiovasculares e cerebrovasculares são as principais causas evitáveis de morte em adultos, acima de 30 anos de idade, que podem ser sanadas e prevenidas por meio de hábitos de vida saudáveis, que comecem na infância Um dos objetivos listados na Declaração é o de prevenir as doenças cardiovasculares e cerebrovasculares, baseando-se em estratégias específicas direcionadas à nutrição e atividade física. Recomenda-se também que a escola seja o foco principal para atingir a criança, a família e a comunidade.

\section{Conclusão}

Os resultados do presente estudo indicaram que os adolescentes obesos apresentam um ou mais indicadores adicionais para risco de doenças cardiovasculares. Entre eles destacaram-se o percentual de gordura corporal e pressão arterial elevados, dislipidemia, presenças de doenças metabólicas na família, sedentarismo e hábitos alimentares inadequados.

Apesar do número reduzido de adolescentes que aceitaram participar da pesquisa e do estudo ter se limitado há apenas três distritos de saúde, este estudo foi relevante no sentido de apontar os indicadores adicionais de risco para doenças cardiovasculares nestes adolescentes obesos e assinalar a necessidade de políticas públicas de 
atenção à saúde visando transformações dos hábitos de vida, por meio de ações e abordagens mais específicas para parcelas da população de maior risco.

O presente estudo foi financiado pela Fundação de Apoio ao Hospital das Clínicas da Universidade Federal de Goiás - FUNDAHC/HC/UFG.

\title{
Risk factors for cardiovascular diseases in obese adolescents from three health districts of goiânia
}

\begin{abstract}
The aim of this research work was to identify and evaluate cardiovascular disease risks for obese adolescents. Items such as arm muscular circumference, tricipital skin fold, lipidemic profile, glycemia, blood pressure, physical activity level, family disease history, and eating habits were evaluated in 42 male and female adolescents aged 13-16. Results indicated that triglycerides, cholesterol, LDL-c, and blood pressure levels were high in $10 \%, 30 \%, 30 \%$ and $21 \%$ of researched adolescents, respectively. Among them, $36 \%$ were low active, $64 \%$ were sedentary and all of them presented inadequate eating habits. The evaluated adolescents presented additional indicators for cardiovascular diseases and they need special care. Keywords: Adolescent - Obesity - Cardiovascular Diseases
\end{abstract}

Factores de riesgo de enfermedades cardiovascular en los adolescentes obesos de tres distritos sanitarios de goiânia

\section{Resumen}

El objetivo de esta investigación fue identificar y evaluar los riesgos de enfermedades cardiovasculares en los adolescentes con exceso de peso corporal. Se evaluó la circunferencia muscular del brazo, el pliegue tricípite, lipidio perfil, nivel de glucosa en sangre, presión arterial, nivel de actividad física, enfermedad en la familia y los hábitos de alimentación de 42 adolescentes de ambos sexos, 13 a 16 años de edad. Los resultados indicaron que los triglicéridos, el colesterol, LDL-C y la presión arterial estaban elevadas en 10\%, 30\%, 30\% y el 21\% de los adolescentes, respectivamente. Entre ellos, el 36\% eran poco activos, $64 \%$ sedentarios todos tenían hábitos alimentarios inadecuados. En conclusión, los adolescentes mostraron indicadores adicionales de enfermedad cardiovascular y, por tanto, necesitan cuidados especiales.

Palabras-clave: Adolescente - Obesidad - Enfermedades Cardiovasculares

\section{Referências}

ABRANTES, M. M.; LAMOUNIER, J. A.; COLOSIMO, E. A. Overweight and obesity prevalence in Northeast and Southeast Regions of Brazil. Rev Assoc Med Bras, São Paulo, v. 49, n. 2, p. 162-9, 2003.

AMERICAN DIABETES ASSOCIATION. Management of Dyslipidemia in Adults whit Diabetes. Diabetes Care, Alexandria, v. 26, Supl1, v. S83-S86, 2003.

ARAUJO, T. L. et al. Análise de indicadores de risco para hipertensão arterial em crianças e adolescentes. Rev Esc Enferm, USP, v. 42, n. 1, p.120-6, 2008.

BRASIL. Ministério da Saúde, Instituto Nacional de Alimentação e Nutrição. Pesquisa nacional sobre saúde e nutrição: condições nutricionais da população brasileira. INAN. Brasília (DF); 1991.

CENTRO DE ESTUDOS DO LABORATÓRIO DE APTIDÃO FÍSICA DE SÃO CAETANO DO SUL (CELAFISCS). Questionário atividade física - youth. Rbs, 2005. Disponível em: http://www.celafiscs.org.br/downloads/QuestionarioAF-Crianca-Adolescente.pdf. Acesso em: 05 de ago. de 2005. 
CENTRO DE ESTUDOS DO LABORATÓRIO DE APTIDÃO FÍSICA DE SÃO CAETANO DO SUL (CELAFISCS). Classificação do nivel de atividade física IPAQ. Disponível em: http://www.celafiscs.org.br/downloads/Classificacaoo-Nive1AF-IPAQ2007.pdf. Acesso em: 05 de ago. de 2006.

COELHO, V. G. et al. Perfil Lipídico e fatores de risco para doenças cardiovasculares em estudantes de medicina. Arq Bras Cardiol, Rio de Janeiro, v. 85, n. 1, p. 57-62, 2005. Disponível em: http://www.arquivosonline.com.br/2005/8501/8501011.pdf. Acesso em 05 de abr. 2009.

DÂMASO, A. Obesidade. Rio de Janeiro: Medsi, 2003.

DENADAI, R. C. et al. Efeitos do exercício moderado e da orientação nutricional sobre a composição corporal de adolescentes obesos avaliados por densitometria óssea (DEXA). Rev Paul Educ Fis, São Paulo, v. 12, n. 2, p. 210-18, 1998.

FERNANDES, R. A. et al. Utilização do Índice de Massa Corporal e dobra da cutânea tricipital como indicadores de adiposidade corporal. Rev da Educação Física, Maringá, v. 18, n. 1, p. 1-7, 2007.

FONTAINE, K. R. et al. Years of life lost due to obesity. JAMA, Chicago, v. 283, n. 2, p. 187- 93, 2003.

FRISANCHO, A. R. Anthropometric standards for the assessment of growth and nutral status. Ann. Arbor, Mich: University of Michigan Press, 1990.

GARROW, J. S.;WEBSTER, J. Quetelet's index $(\mathrm{W} / \mathrm{H})$ as a measure of fatness. Int J Obes., USA, v. 9, p. 147-53, 1985.

GIUGLIANO, R.; MELO, A. L. Diagnóstico de sobrepeso e obesidade em escolares: utilização do Índice de Massa Corporal, segundo padrão internacional. Jornal de Pediatria, Porto Alegre, v. 80, n, 2, p. 12-134, 2004.

GUEDES, D. P. et al. Fatores de risco cardiovasculares em adolescentes: indicadores biológicos e comportamentais. Arq Bras Cardiol, Rio de Janeiro, v. 86, n.6, p. 439-50, 2006.

INTERAMERICAN HEART FOUNDATION. Declaração de Consenso. Declaração sobre a saúde cardiovascular de crianças e jovens. Rio de Janeiro, p. 2-27, 1998.

KRAUSS, R. M. Lipids and lipoproteins in patients whit type 2 diabetes. Diabetes Care, Alexandria, v. 27, n. 6, p.1496- 1504, 2004.

LESSA, I. Doenças crônicas não transmissíveis no Brasil: um desafio para a complexa tarefa da vigilância. Cien Saúde Col, Rio de Janeiro, v. 9, n. 4, p. 931-943, 2004.

MION JUNIOR, D. (Coordenador). V Diretrizes brasileiras de hipertensão arterial. Sociedade Brasileira de Cardiologia. Sociedade Brasileira de Hipertensão. Sociedade Brasileira de Nefrologia, 2006. Disponível em: http://www.sbn.org.br/Diretrizes/V_ Diretrizes_Brasileiras_de_Hipertensao_Arterial.pdf. Acesso em: 5 set. 2007. 
NATIONAL CENTER FOR HEALTH STATISTICS (NCHS). National health and nutrition examination survey. Estados Unidos:, 2002. Disponível em: http:// www.cdc.gov/growthcharts/. Acesso em: 11 mar. 2003.

OLIVEIRA, S. P.; THEBAUD-MONY, A. Hábitos e práticas alimentares em três localidades da cidade de São Paulo (Brasil). Rev Nutr., Campinas, v. 11, n. 1, p. 37- 50, 1998.

PHILIPPI, S. T. et al. Pirâmide alimentar adaptada: guia para escolha dos alimentos. Rev. Nutr, Campinas, v. 12, n. 1, p.65-80, 1999.

PITANGA, FJG; LESSA, I. Prevalência e fatores associados ao sedentarismo no lazer em adultos. Cadernos de Saúde Pública. Rio de Janeiro, v. 21, n.3, p. 870-877, 2005.

RIBEIRO, Q. C. R. et al. Fatores adicionais de risco, cardiovascular associados ao excesso de peso em crianças e adolescentes: o estudo do coração de Belo Horizonte. Arq Bras Cardiol, Rio de Janeiro, v. 86, n. 6, p. 408-18, 2006.

SANTOS, R. D. (coord). III Diretrizes brasileiras sobre dislipidemias e diretriz de prevenção da aterosclerose do departamento de aterosclerose da sociedade brasileira de cardiologia. Arq. Bras. Cardiol. Rio de Janeiro, v.77, suppl.3, p. 1-48. 2001 [online]. Disponível em: http://www.scielo.br/pdf/abc/v77s3/a01v77s3.pdf. Acesso em 10 de abr. 2009.

SANTOS, R. D. et al. Projeto diretrizes da associação médica brasileira. Conselho Federal de Medicina. Revista AMRIGS, Porto Alegre, v. 48, n. 1, p. 43-65, 2004.

SARDINHA, L. B. et al. Receiver operating characteristics analysis of body mass index, triceps skin fold thickness, and arm girth for obesity screening in children and adolescents. Am J Clin Nutr, Davis, v. 70, n. 7, p. 1090-1095, 1999.

SILVA, G. A. P.; BALABAN, G.; MOTTA; M. E. F. A. Prevalência de sobrepeso e obesidade em crianças e adolescentes de diferentes condições socioeconômicas. Rev Bras Saúde Matern Infant, Boa Vista, v. 5, n. 1, p. 53-59, 2005.

SLATER, B. et al.Validation of a semi-quantitative adolescent food frequency questionnaire applied at a public school in São Paulo, Brazil. Eur J Clin Nutr. UK, v. 57, n.5, p. 629-35, 2003.

STABELINI NETO, A. Fatores de risco para aterosclerose relacionado ao nivel de aptidão cardiorrespiratória em adolescentes. 111f. Dissertação [Mestrado em Educação Física] Departamento de Educação Física, Ciências Biológicas. Universidade Federal do Paraná. Curitiba, 2007.

TOJO, R. et al. Hábitos alimentares das crianças em idade pré-escolar e escolar: riscos para a saúde e estratégias para a intervenção. In: Nestlé Nutrition Services. A alimentação da idade pré-escolar até a adolescência. São Paulo, 1995; p.11-13. (Seminário Nestlé Nutrition, 37). 
VIEIRA, V.C. R. et al. Perfil socioeconômico, nutricional e de saúde de adolescentes recém ingressos em uma universidade pública. Rev Nutr., Campinas, v. 15, n.3, p. 273-8, 2002.

VIEIRA, V. C. R. et al. Alterações no padrão alimentar de adolescentes com adequação pôndero-estatural e elevado percentual de gordura corporal. Rev. Bras. Saúde Matern Infant, Boa Vista, v. 5, n. 1, p. 93-102, 2005.

WANG, Y.; MONTEIRO, C. A.; POPKIN, B. M. Trends of obesity and underweight in older children and adolescents in the United States, Brazil, China, and Russia. Am J Clin Nutr., Davis, v. 75, n. 6, p. 971- 7, 2002.

Recebido em: 23 de abril de 2009

Revisado em: 23 de abril de 2009

Aprovado em: 27 de abril de 2009

\section{Endereço para correspondência}

mssilva@fanut.ufg.br. 